\title{
CRKL overexpression promotes cell proliferation and inhibits apoptosis in endometrial carcinoma
}

\author{
LE CAI, HE WANG and QING YANG \\ Department of Gynecology and Obstetrics, Shengjing Hospital of China Medical University, \\ Shenyang, Liaoning 110004, P.R. China
}

Received March 15, 2015; Accepted July 1, 2016

DOI: $10.3892 / \mathrm{ol} .2016 .5394$

\begin{abstract}
The v-Crk avian sarcoma virus CT10 oncogene homolog-like (CRKL) protein is important in cancer progression. However, its expression pattern and biological roles in human endometrial carcinoma remain unexplored. The potential mechanism of CRKL-induced cancer progression is still unclear. The present study aimed to explore the expression pattern and biological roles of CRKL in human endometrial carcinoma. Using immunohistochemistry, it was observed that the CRKL protein was overexpressed in 50.5\% (44/87) of endometrial carcinoma tissues. Plasmid transfection of CRKL into Ishikawa cells was performed, and CRKL overexpression promoted cell proliferation, colony formation and cell cycle transition in the transfected cells. In addition, CRKL overexpression inhibited cell apoptosis in Ishikawa cells treated with cisplatin, with decreased caspase-3 and caspase-9 cleavage. Further analysis revealed that CRKL upregulated the expression of cyclin D1, cyclin E, B cell lymphoma (Bcl)-2 and survivin, and downregulated $\mathrm{Bcl}-2$ associated $\mathrm{X}$ protein expression. In conclusion, the present study demonstrated that CRKL overexpression in endometrial carcinoma contributes to malignant cell growth and resistance to apoptosis, possibly through Bcl-2.
\end{abstract}

\section{Introduction}

Endometrial carcinoma is one of the most common gynecological malignancies, and its incidence is rising (1). Despite improved surgical treatment and the development of adjuvant therapy, the prognosis of endometrial carcinoma has not improved significantly $(2,3)$. Thus, it is important to identify molecular mediators conferring the malignant potential to endometrial carcinoma cells that may be used as tumor markers for predicting the risk of endometrial carcinoma progression (4-6).

Correspondence to: Professor Qing Yang, Department of Gynecology and Obstetrics, Shengjing Hospital of China Medical University, 36 Sanhao Street, Heping, Shenyang, Liaoning 110004, P.R. China

E-mail: yangqingcmu@163.com

Key words: CRKL, endometrial carcinoma, proliferation, Bcl-2
V-Crk avian sarcoma virus CT10 oncogene homolog-like (CRKL) is a member of the CRK family of adapter proteins, which have a variety of biological roles, including cell proliferation, adhesion and migration (7). CRKL was considered to be a key substrate of the break point cluster-Abelson murine leukemia viral oncogene homolog 1 fusion protein in chronic myeloid leukemia (8-10). The CRKL protein has been reported to be upregulated in several malignant cancers (11). CRKL amplification and protein overexpression was observed in lung cancer, pancreatic cancer and colorectal cancer (12-14). In addition, CRKL was demonstrated to facilitate cell malignant invasion, and correlated with poor patient prognosis $(15,16)$. Taken together, these findings suggest that CRKL may serve as an important oncoprotein in cancer development. However, its expression pattern and biological roles in endometrial carcinoma remain unexplored.

In the present study, endogenous CRKL protein expression was examined in 87 endometrial carcinoma specimens. In addition, CRKL expression was upregulated in the Ishikawa cell line, and its effect on cell proliferation and apoptosis was examined. Furthermore, the molecular signaling pathways underlying the biological effects of CRKL were investigated.

\section{Materials and methods}

Patients and specimens. The study protocol was approved by the Institutional Review Board of Shengjing Hospital of China Medical University (Shenyang, China) and written informed consent was obtained from all patients. Primary tumor specimens were obtained from patients diagnosed with endometrioid adenocarcinoma who underwent resection in the First Affiliated Hospital (Shenyang, China) and the Shengjing Hospital of China Medical University between January 2008 and December 2010. The histological diagnosis was evaluated for sections stained with hematoxylin and eosin according to the World Health Organization classification guidelines (17). Clinical and histopathological data were obtained from medical records.

Immunohistochemistry. Surgically excised tumor specimens were fixed with $10 \%$ neutral formalin and embedded in paraffin, and $4-\mu$ m-thick sections were prepared. Immunostaining was performed using the avidin-biotin-peroxidase complex method (Ultra Sensitive $^{\mathrm{TM}}$; Maixin, Fuzhou, China). The sections were 
deparaffinized in xylene, rehydrated with graded alcohol, and then boiled in $0.01 \mathrm{M}$ citrate buffer ( $\mathrm{pH}$ 6.0) for $2 \mathrm{~min}$ in an autoclave. Hydrogen peroxide $(0.3 \%)$ was applied to block the endogenous peroxide activity, and the sections were then incubated with normal goat serum (Invitrogen; Thermo Fisher Scientific, Inc., Waltham, MA, USA) to reduce nonspecific binding. Tissue sections were incubated with an anti-CRKL rabbit polyclonal antibody (1:400; cat. no. ABC242; EMD Millipore, Billerica, MA, USA) at $4^{\circ} \mathrm{C}$ overnight. Rabbit immunoglobulin (Ig) (1:300; cat. no. HPA009178; Sigma-Aldrich, St. Louis, MO, USA) was used as a negative control, by applying the same concentration as that used for the above antigen-specific antibody. Biotinylated goat anti-rabbit serum IgG (1:200; cat. no. A0545; Sigma-Aldrich) was used applied as the secondary antibody at room temperature for $10 \mathrm{~min}$. Upon washing with phosphate-buffered saline (PBS), the sections were incubated with streptavidin-biotin conjugated with horseradish peroxidase, and the peroxidase reaction was developed with 3,3'-diaminobenzidine tetrahydrochloride. Counterstaining with hematoxylin was performed, and the sections were dehydrated in ethanol prior to mounting.

Two independent blinded investigators examined all tumor slides randomly. Five views were examined per slide, and 100 cells were observed per view at x400 magnification. Immunostaining of CRKL was scored on a semiquantitative scale by evaluating the staining intensity and the percentage of positively stained tumor cells. Cytoplasmic and nuclear immunostaining in tumor cells was considered as positive staining. In total, 400 tumor cells were counted, and the percentage of positively stained cells was calculated. The intensity of CRKL staining was scored as 0 (no signal), 1 (moderate) and 2 (strong). The percentage scores were assigned as 1 (1-25\%), 2 (26-50\%), $3(51-75 \%)$ and $4(76-100 \%)$. The scores of each tumor sample were multiplied to obtain a final score of 0-8. Tumor samples scoring 4-8 were considered to exhibit CRKL overexpression.

Cell culture and transfection. The Ishikawa cell line was obtained from the American Type Culture Collection (Manassas, VA, USA). Cells were cultured in Dulbecco's modified Eagle medium (Invitrogen; Thermo Fisher Scientific, Inc.) containing $10 \%$ fetal calf serum (Invitrogen; Thermo Fisher Scientific, Inc.), $100 \mathrm{IU} / \mathrm{ml}$ penicillin (Sigma-Aldrich) and $100 \mu \mathrm{g} / \mathrm{ml}$ streptomycin (Sigma-Aldrich) at $37^{\circ} \mathrm{C}$ in an atmosphere of $5 \% \mathrm{CO}_{2}$. Cells were grown on sterilized culture dishes and were passaged every 2 days with $0.25 \%$ trypsin (Invitrogen; Thermo Fisher Scientific, Inc.).

The pCMV6-CRKL plasmid was purchased from OriGene Technologies, Inc. (Rockville, MD, USA), and was transfected into the cells using Lipofectamine 2000 reagent (Invitrogen; Thermo Fisher Scientific, Inc.). The pCMV6 empty vector was used as a negative control. Cells were harvested $48 \mathrm{~h}$ after transfection and subjected to various analyses.

Reverse transcription-quantitative polymerase chain reaction $(R T-q P C R)$. RT-qPCR was performed using SYBR ${ }^{\circledR}$ Green PCR Master Mix (Applied Biosystems; Thermo Fisher Scientific, Inc.) in a total volume of $20 \mu \mathrm{l}$ on a 7500 Real-Time PCR System (Applied Biosystems; Thermo Fisher Scientific, Inc.) as follows: $95^{\circ} \mathrm{C}$ for $30 \mathrm{sec}$, and 40 cycles of $95^{\circ} \mathrm{C}$ for $5 \mathrm{sec}$ and $60^{\circ} \mathrm{C}$ for $30 \mathrm{sec}$. A dissociation step was performed to generate
Table I. Distribution of CRKL status in endometrial carcinoma according to clinicopathological characteristics.

\begin{tabular}{lcccc}
\hline Characteristics & $\begin{array}{c}\text { No. of } \\
\text { patients }\end{array}$ & $\begin{array}{c}\text { CRKLweak/ } \\
\text { negative }\end{array}$ & $\begin{array}{c}\text { CRKL } \\
\text { positive }\end{array}$ & P-value \\
\hline Age, years & & & & 0.7614 \\
$<60$ & 56 & 27 & 29 & \\
$\geq 60$ & 31 & 16 & 15 & \\
Grade & & & & 0.0409 \\
1 & 37 & 23 & 14 & \\
$2+3$ & 50 & 20 & 30 & \\
T stage & & & & 0.7324 \\
T1a & 9 & 5 & 4 & \\
T1b & 43 & 23 & 20 & \\
T1c & 29 & 13 & 16 & \\
T2-3 & 6 & 2 & 4 & \\
FIGO stage & & & & 0.7001 \\
I & 59 & 30 & 29 & \\
II+III & 28 & 13 & 15 & \\
\hline
\end{tabular}

CRKL, v-Crk avian sarcoma virus CT10 oncogene homolog-like; FIGO, International Federation of Gynecology and Obstetrics.

a melting curve to confirm the specificity of the amplification. $\beta$-actin was used as the reference gene. The relative levels of gene expression were represented as $\Delta \mathrm{Cq}=\mathrm{Cq}$ gene - Cq reference, and the fold-change of gene expression was calculated by the $2^{-\triangle \Delta C q}$ method (18). Experiments were conducted in triplicate. The primer sequences were as follows: CRKL forward, 5'-CCTTTGCCATCCACACAGAAT-3' and reverse, 5'-TTT CACGATGTCACCAACCTCTA-3'; and $\beta$-actin forward, 5'-ATAGCACAGCCTGGATAGCAACGTAC-3' and reverse, 5'-CACCTTCTACAATGAGCTGCGTGTG-3'.

Western blot analysis. Total proteins from cells were extracted in lysis buffer (Pierce; Thermo Fisher Scientific, Inc.) and quantified using the Bradford method. Proteins (50 $\mu \mathrm{g})$ were separated by $10 \%$ sodium dodecyl sulfate-polyacrylamide gel electrophoresis. Samples were transferred to polyvinylidene fluoride membranes (EMD Millipore) and incubated overnight at $4^{\circ} \mathrm{C}$ with antibodies against CRKL (rabbit polyclonal; 1:1,000; cat. no. ABC242; EMD Millipore), B cell lymphoma (Bcl)-2 (rabbit monoclonal; 1:1,000; cat. no. 3948; Cell Signaling Technology, Inc., Danvers, MA, USA), Bcl-2 associated $\mathrm{X}$ protein (Bax) (rabbit monoclonal; 1:1,000; cat. no. 4223; Cell Signaling Technology, Inc.), survivin (rabbit monoclonal; 1:1,000; cat. no. 2808; Cell Signaling Technology, Inc.), caspase-3 (rabbit monoclonal; 1:1,000; cat. no. 9665; Cell Signaling Technology, Inc.), cleaved caspase-3 (rabbit monoclonal; 1:1,000; cat. no. 9664; Cell Signaling Technology, Inc.), caspase-9 (rabbit monoclonal; 1:1,000; cat. no. 9502; Cell Signaling Technology, Inc.), cleaved caspase-9 (rabbit monoclonal; 1:1,000; cat. no. 9501; Cell Signaling Technology, Inc.), cyclin D1 (rabbit monoclonal; 1:1,000; cat. no. 2978; Cell Signaling Technology, Inc.), cyclin E (rabbit monoclonal; 1:1,000; cat. no. 20808; 

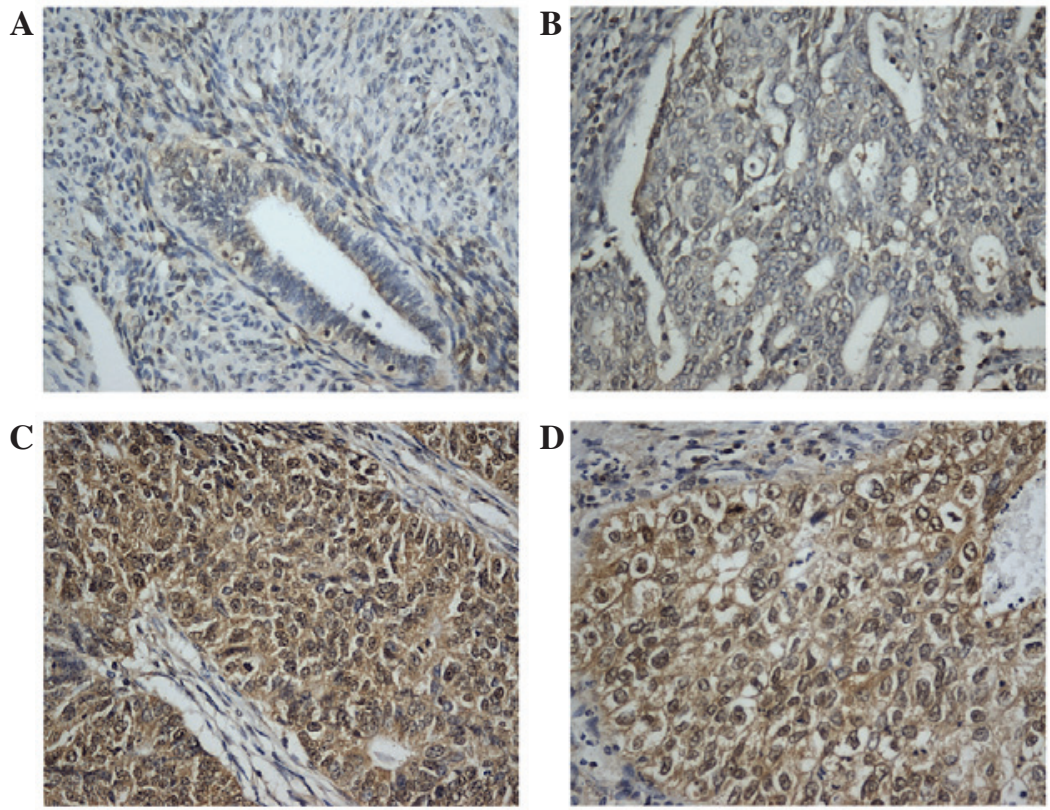

Figure 1. Expression of CRKL protein in endometrial carcinoma tissues. (A) Weak CRKL expression in normal endometrial tissue. (B) Negative CRKL expression in a case of grade 1 endometrial carcinoma. (C) Positive staining of CRKL in a case of grade 1 endometrial carcinoma. (D) Positive CRKL expression in a case of grade 2 endometrial carcinoma. Magnification, x400. CRKL, v-Crk avian sarcoma virus CT10 oncogene homolog-like.

Cell Signaling Technology, Inc.) and glyceraldehyde 3-phosphate dehydrogenase (GAPDH) (rabbit polyclonal; 1:1,000; cat. no. sc-25778; Santa Cruz Biotechnology, Inc., Dallas, TX, USA). After incubation with peroxidase-coupled monoclonal mouse anti-rabbit IgG (1:1,000; cat. no. 5127; Cell Signaling Technology, Inc.) at $37^{\circ} \mathrm{C}$ for $2 \mathrm{~h}$, the bound proteins were visualized by enhanced chemiluminescence (Pierce; Thermo Fisher Scientific, Inc.) and detected using an imaging system (DNR Bio-Imaging Systems Ltd., Jerusalem, Israel). Relative protein levels were quantified using GAPDH as the loading control.

Colony formation and 3-(4,5-dimethylthiazol-2-yl)-2,5-diphenyltetrazolium bromide (MTT) assays. For the colony formation assay, cells were transfected with the aforementioned plasmids for $48 \mathrm{~h}$, and then plated into three $6-\mathrm{cm}$ cell culture dishes $(2,000$ cells/dish). Cells were incubated for 12 days in medium containing $10 \%$ fetal bovine serum. Plates were next washed with PBS and stained with Giemsa. The number of colonies containing $>50$ cells was counted manually using a microscope.

For the MTT assay, $24 \mathrm{~h}$ after transfection, cells were plated in 96-well plates at a density of $~ 3,000$ cells/well, and were cultured for 5 days. For quantitation of cell viability, $20 \mu \mathrm{l}$ of $5 \mathrm{mg} / \mathrm{ml} \mathrm{MTT}$ (thiazolyl blue) solution was added to each well, and incubated for $4 \mathrm{~h}$ at $37^{\circ} \mathrm{C}$. Subsequently, the medium was removed from each well, and the resulting MTT formazan was solubilized in $150 \mu \mathrm{l}$ of dimethyl sulfoxide. Each solution was measured spectrophotometrically at $490 \mathrm{~nm}$.

Cell cycle and apoptosis analysis. Cells (100,000) were seeded into $6-\mathrm{cm}$ tissue culture dishes, and $12 \mathrm{~h}$ later were transfected with the indicated amounts of plasmid (1.2 $\mu \mathrm{g} / \mathrm{dish})$. After $48 \mathrm{~h}$, cells were harvested, fixed in $1 \%$ paraformaldehyde, washed with PBS and stained in $5 \mathrm{mg} / \mathrm{ml}$ propidium iodide (PI) in PBS supplemented with RNase A (Roche Diagnostics, Indianapolis, IN, USA) for $30 \mathrm{~min}$ at room temperature. Data were collected using a BD system (BD Biosciences, Franklin Lakes, NJ, USA).

Cell apoptosis detection was performed with Annexin V/PI double staining. Briefly, $48 \mathrm{~h}$ after transfection, cells were harvested by $0.25 \%$ trypsin, washed twice with chilled PBS and resuspended in $250 \mu \mathrm{l}$ of binding buffer. Staining solution containing Annexin V/fluorescein isothiocyanate and PI was added to the cell suspension. After incubation in the dark for $30 \mathrm{~min}$, cells were analyzed by FACSCalibur flow cytometer (BD Biosciences).

Statistical analysis. SPSS version 11.5 (SPSS, Inc., Chicago, IL, USA) was used for all statistical analyses. The $\chi^{2}$ test was used to examine possible correlations between CRKL expression and clinicopathological factors. The Student's $t$-test was used to compare densitometry data between the control and CRKL-transfected cells. All P-values are based on a two-sided statistical analysis, and $\mathrm{P}<0.05$ was considered to indicate a statistically significant difference.

\section{Results}

Expression of CRKL in human endometrial carcinoma. CRKL immunostaining was examined in 87 cases of endometrial carcinoma. In normal endometrial tissues, stromal and glandular tissues exhibited weak or negative staining (Fig. 1A). In total, 44 of $87(50.5 \%)$ primary endometrial cancers displayed positive CRKL immunoreactivity, which was located in the nuclear and cytoplasmic compartment of the carcinoma cells (Fig. 1B-D). The association of CRKL overexpression with clinicopathological characteristics is listed in Table I. High CRKL immunostaining score in the 
A

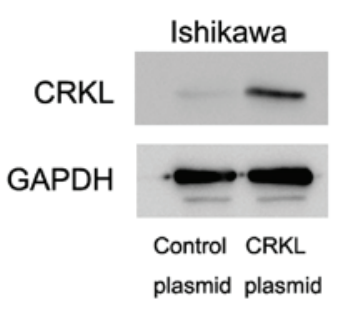

C

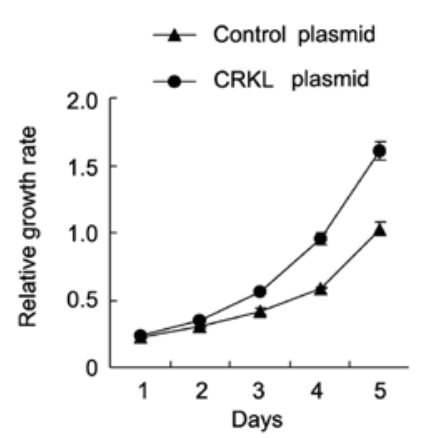

$\mathbf{E}$

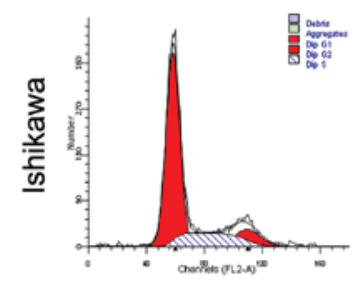

Control

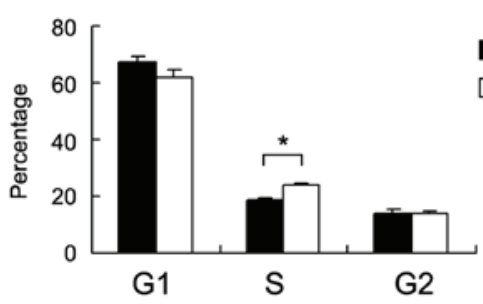

Figure 2. CRKL transfection promotes cell proliferation and cell cycle in the Ishikawa endometrial carcinoma cell line. (A) Western blot analysis demonstrated that CRKL transfection in the Ishikawa cell line upregulated CRKL protein expression. (B) Reverse transcription-quantitative polymerase chain reaction analysis demonstrated that CRKL transfection in the Ishikawa cel line upregulated CRKL messenger RNA expression. " $\mathrm{P}<0.001$ vs. control. (C) 3-(4,5-dimethylthiazol-2-yl)-2,5-diphenyltetrazolium bromide assay revealed that CRKL transfection upregulated cell proliferation. (D) Colony formation assay revealed that CRKL transfection increased the colony formation ability of Ishikawa cells. ${ }^{*} \mathrm{P}<0.001$ vs. control. (E) Flow cytometry analysis demonstrated that CRKL transfection facilitated G1-S cell cycle transition. ${ }^{*} \mathrm{P}=0.023$ vs. control. CRKL, v-Crk avian sarcoma virus CT10 oncogene homolog-like; GAPDH, glyceraldehyde 3-phosphate dehydrogenase; mRNA, messenger RNA; Dip, diploid.

primary endometrial carcinoma was significantly correlated with advanced tumor grade (grade $2+3$ vs. grade 1 , $\mathrm{P}=0.0409)$. CRKL expression was relatively higher in tumors with advanced T stage (T2-3, 66.6\%) compared with tumors with lower T stage (T1a, T1b and T1c, 49.3\%), which did not reach statistical significance. No significant correlation was observed between CRKL level and other parameters such as International Federation of Gynecology and Obstetrics stage $(\mathrm{P}=0.7001)$ or patient age $(\mathrm{P}=0.7614)$.
A

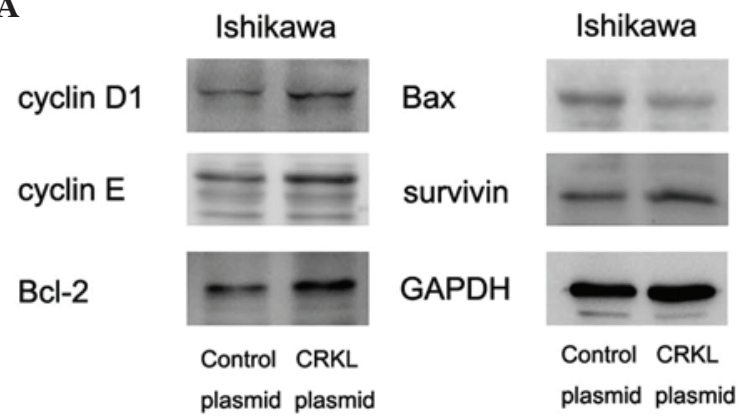

B Cisplatin $(1 \mu \mathrm{M})$

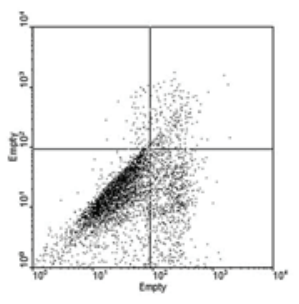

Control plasmid

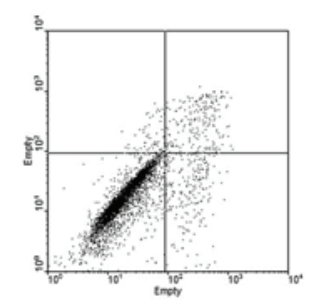

CRKL plasmid
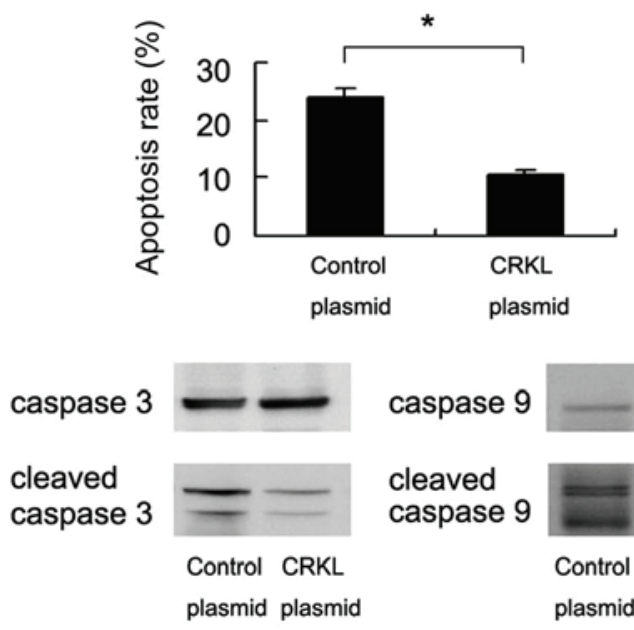

caspase 9

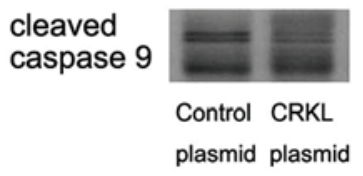

Figure 3. CRKL inhibits apoptosis of Ishikawa cells. (A) CRKL transfection upregulated the protein expression of cyclin D1, cyclin E, Bcl-2 and survivin, and downregulated that of $\mathrm{Bcl}-2$ associated $\mathrm{X}$ protein. (B) CRKL transfection was performed in Ishikawa cells, and cisplatin treatment ( $1 \mu \mathrm{M}$ for $24 \mathrm{~h}$ ) was applied $48 \mathrm{~h}$ after transfection. The rate of cell apoptosis was significantly decreased in CRKL-overexpressing cells. The levels of caspase-3 cleavage and caspase- 9 cleavage were also decreased. ${ }^{*} \mathrm{P}<0.001$ vs. control. CRKL, v-Crk avian sarcoma virus CT10 oncogene homolog-like; GAPDH, glyceraldehyde 3-phosphate dehydrogenase; Bcl-2, B cell lymphoma-2; Bax, Bcl-2 associated $\mathrm{X}$ protein

CRKL promotes Ishikawa cell proliferation and upregulates cell cycle proteins. To determine the biological roles of CRKL in endometrial cancer, plasmid transfection was performed in the Ishikawa cell line. As shown in Fig. 2A and B, CRKL plasmid transfection significantly upregulated CRKL protein and messenger RNA expression. MTT assay revealed that CRKL upregulation increased the cell proliferation rate (Fig. 2C). Colony formation assay was also conducted, as shown in Fig. 2D, and the results revealed that CRKL transfection significantly increased the colony number of Ishikawa cells $(83 \pm 9$ colonies for empty vector-transfected vs. $156 \pm 12$ colonies for $\mathrm{CRKL}$ plasmid-transfected cells, $\mathrm{P}<0.05$ ). 
Cell cycle analysis was performed, and it was observed that CRKL transfection increased the percentage of cells in the $\mathrm{S}$ phase and decreased the percentage of cells in the G1 phase, thus suggesting that CRKL facilitated the G1-S cell cycle transition (Fig. 2E). Accordingly, CRKL transfection upregulated cyclin D1 and cyclin E expression (Fig. 3A).

CRKL inhibits Ishikawa cell apoptosis and upregulates Bcl-2 and survivin. In order to investigate the association between CRKL and apoptosis in endometrial carcinoma cells, control cells and CRKL-transfected cells were treated with cisplatin (1 $\mu \mathrm{M}, 24 \mathrm{~h})$ and subjected to cell apoptosis analysis. Compared with the control group, the rate of cell apoptosis in CRKL-overexpressing Ishikawa cells decreased significantly (empty vector vs. CRKL plasmid, $23.8 \pm 1.7$ vs. $10.3 \pm 1.2 \%$, respectively, $\mathrm{P}=0.009$ ) (Fig. 3B). Accordingly, the levels of caspase-3 and caspase-9 cleavage decreased upon CRKL upregulation. The level of apoptosis-related proteins was also examined, and CRKL transfection was observed to upregulate $\mathrm{Bcl}-2$ and survivin expression in the Ishikawa cell line. By contrast, the level of Bax was downregulated following CRKL transfection (Fig. 3A).

\section{Discussion}

Endometrial carcinoma is one of the most common gynaecological cancers worldwide $(19,20)$. Although previous studies have reported that multiple aberrantly expressed genes in endometrial carcinoma could contribute to the malignant behavior $(15,21-24)$, novel markers that are able to identify tumor progression and predict the aggressive phenotype are still urgently required. Upregulation of CRKL expression has been implicated in several human cancers, including breast cancer, lung cancer and pancreatic cancer $(15,16,25,26)$, where it leads to increased proliferation and invasion. However, its biological roles and clinical significance in human endometrial carcinoma remain unexplored. The present study examined CRKL protein expression in 87 cases of endometrial carcinoma, and CRKL overexpression was observed in $50.5 \%$ of cancer tissues. CRKL overexpression positively correlated with advanced tumor grade, thus suggesting its association with malignant phenotype. The current data is in accordance with previous reports that confirmed CRKL as an oncogene overexpressed in human endometrial carcinomas.

There is a growing body of evidence suggesting that CRKL is involved in carcinogenesis of solid tumors by regulating their biological behavior, including proliferation, differentiation and invasion (27-29). In order to assess the function of CRKL in endometrial carcinoma progression, a CRKL-expression plasmid was employed in the Ishikawa cell line. The results demonstrated that transient transfection of the above CRKL plasmid caused an obvious increase in the proliferation rate and colony formation ability of Ishikawa cells. In addition, cell cycle analysis revealed that the percentage of cells in the $\mathrm{S}$ phase was increased in CRKL-transfected cells, thus suggesting that CRKL promotes cell growth through the G1-S transition. The present study also examined cell cycle-related factors, and observed that CRKL caused upregulation of cyclin E and cyclin D1, which control cell cycle progression through the restriction point at the G1-S phase $(26,30)$. Previous reports demonstrated that cyclin D1 and cyclin E were overexpressed in endometrial carcinoma and were important in cancer progression $(31,32)$. The current results were in accordance with their role in cell cycle control.

The association of CRKL with apoptosis is not well characterized. In the present study, CRKL overexpression led to decreased cisplatin-induced apoptosis in Ishikawa cells. CRKL transfection also caused decreased caspase- 3 and caspase-9 cleavage, which suggests that CRKL may function as an important modifier of the apoptosis process in endometrial carcinoma cells. Regulation of cancer cell apoptosis involves the interaction of multiple genes. In the current study, the change in the levels of Bcl-2 and survivin in endometrial carcinoma cells upon CRKL overexpression was examined, and it was observed that CRKL overexpression increased the levels of Bcl-2 and survivin, both of which have been reported to be involved in apoptosis inhibition in numerous cancers, including endometrial carcinoma (33-36). The present data strongly support the role of CRKL in regulating cell apoptosis through Bcl-2 and survivin.

In conclusion, CRKL is overexpressed in human endometrial carcinomas and correlates with advanced tumor grade. CRKL promotes tumor proliferation through regulation of cyclin proteins, and inhibits apoptosis through Bcl-2 and survivin upregulation. CRKL may serve as a novel therapeutic target for endometrial carcinoma.

\section{Acknowledgements}

This study was supported by the Shenyang Science and Technology Project (grant no. F15-139-9-33).

\section{References}

1. Siegel R, Desantis C and Jemal A: Colorectal cancer statistics, 2014. CA Cancer J Clin 64: 104-117, 2014.

2. Sivridis E, Giatromanolaki A, Gatter KC, Harris AL and Koukourakis MI; Tumor and Angiogenesis Research Group: Association of hypoxia-inducible factors 1alpha and 2alpha with activated angiogenic pathways and prognosis in patients with endometrial carcinoma. Cancer 95: 1055-1063, 2002.

3. Sakuragi N, Ohkouchi T, Hareyama H, Ikeda K, Watari H, Fujimoto T, Kuwabara M, Yamamoto R, Sagawa T, Fujino T and Fujimoto S: Bcl-2 expression and prognosis of patients with endometrial carcinoma. Int J Cancer 79: 153-158, 1998.

4. Wen SY, Zhang LN, Yang XM, Zhang YL, Ma L, Ge QL, Jiang SH, Zhu XL, Xu W, Ding WJ, et al: LRG1 is an independent prognostic factor for endometrial carcinoma. Tumour Biol 35: 7125-7133, 2014.

5. Saarelainen SK, Staff S, Peltonen N, Lehtimäki T, Isola J, Kujala PM, Vuento MH and Mäenpää JU: Endoglin, VEGF and its receptors in predicting metastases in endometrial carcinoma. Tumour Biol 35: 4651-4657, 2014.

6. Honkavuori-Toivola M, Talvensaari-Mattila A, Soini Y, Turpeenniemi-Hujanen T and Santala M: Immunoreactivity for TIMP-2 is associated with a favorable prognosis in endometrial carcinoma. Tumour Biol 33: 935-941, 2012.

7. Rhodes J, York RD, Tara D, Tajinda K and Druker BJ: CrkL functions as a nuclear adaptor and transcriptional activator in Bcr-Abl-expressing cells. Exp Hematol 28: 305-310, 2000.

8. Feller SM: Crk family adaptors-signalling complex formation and biological roles. Oncogene 20: 6348-6371, 2001.

9. Fidler IJ and Kripke ML: Genomic analysis of primary tumors does not address the prevalence of metastatic cells in the population. Nat Genet 34: 23, 2003.

10. Hoeve J, Morris C, Heisterkamp N and Groffen J: Isolation and chromosomal localization of CRKL, a human crk-like gene. Oncogene 8: 2469-2474, 1993. 
11. ten Hoeve J, Kaartinen V, Fioretos T, Haataja L, Voncken JW, Heisterkamp N and Groffen J: Cellular interactions of CRKL, and SH2-SH3 adaptor protein. Cancer Res 54: 2563-2567, 1994.

12. Beroukhim R, Mermel CH, Porter D, Wei G, Raychaudhuri S, Donovan J, Barretina J, Boehm JS, Dobson J, Urashima M, et al: The landscape of somatic copy-number alteration across human cancers. Nature 463: 899-905, 2010.

13. Senechal K, Halpern J and Sawyers CL: The CRKL adaptor protein transforms fibroblasts and functions in transformation by the BCR-ABL oncogene. J Biol Chem 271: 23255-23261, 1996.

14. van't Veer LJ, Dai H, van de Vijver MJ, He YD, Hart AA, Mao M, Peterse HL, van der Kooy K, Marton MJ, Witteveen AT, et al: Gene expression profiling predicts clinical outcome of breast cancer. Nature 415: 530-536, 2002.

15. Lin F, Chengyao X, Qingchang L, Qianze D, Enhua W and Yan W: CRKL promotes lung cancer cell invasion through ERK-MMP9 pathway. Mol Carcinog (54 Suppl 1): E35-E44, 2015.

16. Wang Y, Dong QZ, Fu L, Stoecker M, Wang E and Wang EH Overexpression of CRKL correlates with poor prognosis and cell proliferation in non-small cell lung cancer. Mol Carcinog 52: 890-899, 2013

17. Ali RH and Rouzbahman M: Endometrial stromal tumours revisited: An update based on the 2014 WHO classification. J Clin Pathol 68: 325-332, 2015.

18. Cikos S, Bukovská A and Koppel J: Relative quantification of mRNA: Comparison of methods currently used for real-time PCR data analysis. BMC Mol Biol 8: 113, 2007.

19. Buhtoiarova TN, Brenner CA and Singh M: Endometrial carcinoma: Role of current and emerging biomarkers in resolving persistent clinical dilemmas. Am J Clin Pathol 145: 8-21, 2016.

20. Zhang L, Yan L, Cao M, Zhang H, Li C, Bai Y, Yu P, Li M and Zhao X: SPAG9 promotes endometrial carcinoma cell invasion through regulation of genes related to the epithelial-mesenchymal transition. Eur J Gynaecol Oncol 37: 312-319, 2016.

21. Lian X, Jiao Y, Yang Y, Wang Z, Xuan Q, Liu H, Lu S, Wang Z, Liu Y, Li S, et al: CrkL regulates SDF-1-induced breast cancer biology through balancing Erk1/2 and PI3K/Akt pathways. Med Oncol 32: $411,2015$.

22. Lv S, Qin J, Yi R, Coreman M, Shi R, Kang H and Yao C: CrkL efficiently mediates cell proliferation, migration, and invasion induced by TGF- $\beta$ pathway in glioblastoma. J Mol Neurosci 51: 1046-1051, 2013

23. Nosaka Y, Arai A, Miyasaka N and Miura O: CrkL mediates Ras-dependent activation of the Raf/ERK pathway through the guanine nucleotide exchange factor $\mathrm{C} 3 \mathrm{G}$ in hematopoietic cells stimulated with erythropoietin or interleukin-3. J Biol Chem 274 30154-30162, 1999.

24. Segovis CM, Schoon RA, Dick CJ, Nacusi LP, Leibson PJ and Billadeau DD: PI3K links NKG2D signaling to a CrkL pathway involved in natural killer cell adhesion, polarity, and granule secretion. J Immunol 182: 6933-6942, 2009.
25. Zhao T, Miao Z, Wang Z, Xu Y, Wu J, Liu X, You Y and Li J: Overexpression of CRKL correlates with malignant cell proliferation in breast cancer. Tumour Biol 34: 2891-2897, 2013.

26. Fu L, Dong Q, Xie C, Wang Y and Li Q: CRKL protein overexpression enhances cell proliferation and invasion in pancreatic cancer. Tumour Biol 36: 1015-1022, 2015.

27. Singer CF, Hudelist G, Lamm W, Mueller R, Handl C, Kubista E and Czerwenka K: Active (p)CrkL is overexpressed in human malignancies: Potential role as a surrogate parameter for therapeutic tyrosine kinase inhibition. Oncol Rep 15: 353-359, 2006.

28. Kim YH, Kwei KA, Girard L, Salari K, Kao J, Pacyna-Gengelbach M, Wang P, Hernandez-Boussard T, Gazdar AF, Petersen I, et al: Genomic and functional analysis identifies CRKL as an oncogene amplified in lung cancer. Oncogene 29: 1421-1430, 2010.

29. Fathers KE,BellES, RajaduraiCV,CoryS,ZhaoH,Mourskaia A, Zuo D, Madore J, Monast A, Mes-Masson AM, et al: Crk adaptor proteins act as key signaling integrators for breast tumorigenesis. Breast Cancer Res 14: R74, 2012.

30. Wang J, Chen X, Li P, Su L, Yu B, Cai Q, Li J, Yu Y, Liu B and Zhu Z: CRKL promotes cell proliferation in gastric cancer and is negatively regulated by miR-126. Chem Biol Interact 206: 230-238, 2013

31. Ruhul Quddus M, Latkovich P, Castellani WJ, James Sung C, Steinhoff MM, Briggs RC and Miranda RN: Expression of cyclin D1 in normal, metaplastic, hyperplastic endometrium and endometrioid carcinoma suggests a role in endometrial carcinogenesis. Arch Pathol Lab Med 126: 459-463, 2002.

32. Cassia R, Moreno-Bueno G, Rodriguez-Perales S, Hardisson D, Cigudosa JC and Palacios J: Cyclin E gene (CCNE) amplification and hCDC4 mutations in endometrial carcinoma. J Pathol 201: 589-595, 2003.

33. González-Rodilla I, Verna V, Muñoz AB, Estévez J, Boix M and Schneider J: Expression of the apoptosis-related genes Bcl-2 and p53 in clinical samples from endometrial carcinoma patients. Anticancer Res 31: 4191-4193, 2011.

34. Porichi O, Nikolaidou ME, Apostolaki A, Tserkezoglou A, Arnogiannaki N, Kassanos D, Margaritis L and Panotopoulou E: BCL-2, BAX and P53 expression profiles in endometrial carcinoma as studied by real-time PCR and immunohistochemistry. Anticancer Res 29: 3977-3982, 2009.

35. Pallares J, Martinez-Guitarte JL, Dolcet X, Llobet D, Rue M, Palacios J, Prat J and Matias-Guiu X: Survivin expression in endometrial carcinoma: A tissue microarray study with correlation with PTEN and STAT-3. Int J Gynecol Pathol 24: 247-253, 2005

36. Takai N, Miyazaki T, Nishida M, Nasu K and Miyakawa I: Survivin expression correlates with clinical stage, histological grade, invasive behavior and survival rate in endometrial carcinoma. Cancer letters 184: 105-116, 2002. 